\title{
Anomalous behaviour of the UV-optical continuum bands in NGC 5548
}

\author{
M. R. Goad, ${ }^{1 \star}$ C. Knigge ${ }^{2}$ K. T. Korista, ${ }^{3}$ E. Cackett, ${ }^{4}$ K. Horne, ${ }^{5}$ D. A. Starkey, ${ }^{5}$ \\ B. M. Peterson, ${ }^{6,7,8}$ G. De Rosa, ${ }^{7}$, G. A. Kriss, ${ }^{7}$ R. Edelson, ${ }^{9}$ M. Fausnaugh ${ }^{6,10}$ \\ ${ }^{1}$ University of Leicester, Department of Physics and Astronomy, University Road, Leicester LE1 7RH, UK \\ ${ }^{2}$ School of Physics \& Astronomy, University of Southampton, Highfield, Southampton SO17 1BJ, UK \\ ${ }^{3}$ Western Michigan University, Department of Physics, 1120 Everett Tower, Kalamazoo, MI 49008-5252, USA \\ ${ }^{4}$ Wayne State University, Department of Physics and Astronomy, $666 \mathrm{~W}$. Hancock St, Detroit, MI 48201, USA \\ ${ }^{5}$ SUPA Physics and Astronomy, University of St. Andrews, Fife, KY16 9SS Scotland, UK \\ ${ }^{6}$ Department of Astronomy, The Ohio State University, $140 \mathrm{~W} 18$ th Ave, Columbus, OH 43210, USA \\ ${ }^{7}$ Space Telescope Science Institute, 3700 San Martin Drive, Baltimore, MD 21218, USA \\ ${ }^{8}$ Center for Cosmology and AstroParticle Physics, The Ohio State University, 191 West Woodruff Ave, Columbus, OH 43210, USA \\ ${ }^{9}$ University of Maryland, Department of Astronomy, College Park, MD 20742-2421, USA \\ ${ }^{10}$ Department of Physics, Massachussetts Institute of Technology, 77 Massachussetts Avenue, Cambridge, MA 02139-4307, USA
}

Accepted 2019 April 25. Received 2019 April 9; in original form 2018 December 8

\begin{abstract}
During the 2014 HST/Swift and ground-based multi-wavelength monitoring campaign of NGC 5548 (AGN STORM), the UV-optical broad emission lines exhibited anomalous, decorrelated behaviour relative to the far-UV continuum flux variability. Here, we use key diagnostic emission lines (Ly $\alpha$ and He II) for this campaign to infer a proxy for the all important, variable driving EUV continuum incident upon BLR clouds. The inferred driving continuum provides a crucial step towards the recovery of the broad emission line response functions in this AGN. In particular, the ionising continuum seen by the BLR was weaker and softer during the anomalous period than during the first third of the campaign, and apparently less variable than exhibited by the far-UV continuum. We also report the first evidence for anomalous behaviour in the longer wavelength (relative to $\lambda 1157 \AA$ ) continuum bands. This is corroborative evidence that a significant contribution to the variable UV-optical continuum emission arises from a diffuse continuum emanating from the same gas that emits the broad emission lines.
\end{abstract}

Key words: galaxies: active — galaxies: individual (NGC 5548) — galaxies: nuclei - galaxies: Seyfert

\section{INTRODUCTION}

Correlated continuum and broad emission line variability studies (reverberation mapping, hereafter RM) have proven a powerful probe of the central regions of Active Galactic Nuclei (AGN). Following the seminal paper by Blandford \& McKee (1982), it was soon realised that RM could not only reveal the spatial distribution and kinematics of the line emitting gas, but also, with few assumptions, provide an estimate for the mass of the central super-massive black hole (e.g., Peterson et al. 2002, 2014; Bentz et al. 2009, 2010a,b, 2014; Grier et al. 2012; Kaspi et al. 2002, 2007; Pancoast et al. 2012, 2014a,b). This technique has yielded black hole mass estimates for $\approx 60$, mostly nearby and low-luminosity, AGN (see Bentz \& Katz (2015), for details). RM black hole

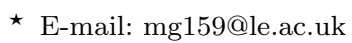

mass determinations are competitive with mass estimates derived using more conventional techniques (e.g., stellar velocity dispersion), while high source luminosities afford access to mass determinations at high redshift. RM is thus important for studying the accretion flow and energy generation mechanism in AGN, and for investigating black hole growth on cosmological timescales (Silk 2013; Silk \& Rees 1998).

While RM was largely developed for broad emission-line variability studies, it has also been successfully applied to the continuum bands alone. Specifically, correlated opticalIR continuum variations (dust reverberation) can provide a robust estimate of the distance to the hot-dust, an upper limit to the BLR outer boundary and an important constraint for models of the BLR. Dust-delay measurements now exist for $\sim$ a dozen nearby AGN (Koshida et al. 2014; Suganuma et al. 2006; Minezaki et al. 2004; Oknyanskij 
\& Horne 2001; Clavel, Wamsteker, \& Glass 1989; references therein). Most recently, inter-band continuum delays at shorter wavelengths have been used to probe the disk radial temperature profile, and if the black hole mass is known, the mass accretion rate through the disk (Collier 2001; Collier \& Peterson 2001; Sergeev et al. 2005; Cackett et al. 2007; Troyer et al. 2016; Edelson et al. 2015, 2017, 2018; Fausnaugh et al. 2016; Starkey et al. 2017; Cackett et al. 2018, McHardy et al. 2018). Thus RM can be used to study the dominant components occupying the central regions of AGN on small (disk), intermediate (BLR), and large (dusty torus) scales, and the relationship(s) between them.

\subsection{The AGN STORM Campaign}

NGC 5548, a nearby Seyfert 1.5, has a well-documented history of short timescale large amplitude continuum and broad emission line variability, and is thus an ideal target for AGN variability campaigns (e.g., Wamsteker et al. 1990; Clavel et al. 1991; Korista et al. 1995; Peterson et al. 2002, and references therein). From 2014 February-July, NGC 5548 was the subject of the most ambitious spectroscopic reverberation mapping campaign to date, the AGN Space Telescope and Optical Reverberation Mapping project (AGN STORM, Peterson PI), with daily monitoring with HST/COS over 179 days, yielding 171 usable epochs. The $H S T / C O S$ program was amply supported by an intensive Swift/XRT/UVOT photometric monitoring campaign (Edelson, PI) and ground-based optical photometry and spectroscopy (De Rosa et al. 2015; Edelson et al. 2015; Fausnaugh et al. 2016, Pei et al. 2017, for details). In addition, 4 Chandra X-ray spectra were obtained, over the time period spanned by the campaign (Mathur et al. 2017).

Key results from AGN STORM include:

(i) identification of a stratified BLR with mean broad emission-line delays spanning $2.5-7$ days with respect to the $\lambda 1157 \AA$ continuum, depending on the emission line (De Rosa et al. 2015). When placed in context of previous intensive monitoring campaigns of this source (e.g., Clavel et al. 1991; Peterson et al. 1991, 2002; Korista et al. 1995; Denney et al. 2009), the measured delays and variability amplitudes (i.e., responsivities) for the strong UV and optical broad emission lines for NGC 5548 during 2014 are significantly smaller, even though the continuum luminosity during 2014 is comparable to that in previous campaigns;

(ii) line of sight velocity versus mean delays indicative of a radially decreasing velocity field (De Rosa et al. 2015);

(iii) a robust detection of X-ray-UV-optical continuum interband delays (Edelson et al. 2015; Fausnaugh et al. 2016, Starkey et al. 2017);

(iv) the $U$ band lag is a factor of $>2$ longer than the surrounding continuum bands $(U V W 1$ and $B)$ and in excess of the delay predicted from fitting a simple disk-reprocessing model to the data. This is a clear indication that diffuse continuum emission (hereafter, DC) from the BLR (Korista \& Goad 2001) affects the continuum interband lags (e.g., Edelson et al. 2018);

(v) the appearance of anomalous behaviour in the broad emission lines starting mid-way through the campaign and lasting $\approx 60$ days (Goad et al. 2016; Pei et al. 2017), as described below. Almost certainly related to this phenomenon is the sudden increase in the soft X-ray emission prior to the onset of the anomaly followed by a more gradual decline (Mathur et al. 2017).

Detailed studies of the time and wavelength-dependent continuum behaviour have the potential to reveal the nature of the central engines of AGN: the accretion structure, the means of energy propagation within the accretion structure, and the origin of the ubiquitous luminous flux variability in AGN. Significantly, the measured inter-band continuum variations during the AGN STORM campaign were characterised by increased delays and reduced amplitude variations at longer wavelengths and, though qualitatively consistent with a simple thin-disk reprocessing scenario, imply a disk size in NGC 5548 which is a factor few too large for its luminosity (Edelson et al. 2015; Fausnaugh et al. 2016; Starkey et al. 2017), similar to that found from disk reverberation mapping studies of other nearby AGN (Cackett et al. 2007, 2018; Troyer et al. 2016). Quasar micro-lensing studies also point to larger than expected disk sizes (Poindexter et al. 2008; Morgan et al. 2010; Mosquera et al. 2013). While alternate explanations abound (e.g., Gardner \& Done 2017; Dexter \& Agol 2011), interpretation of the measured delay signatures first requires careful accounting of other known variable contributions to the observed continuum, such as from the BLR (see e.g., Korista \& Goad 2001; hereafter KG01).

Of particular interest here is the appearance midway through the campaign of anomalous behaviour in the $\mathrm{UV} /$ optical broad emission lines relative to the observed farUV continuum (Figure 1, $\S 2.1$; see also Goad et al. 2016; Pei et al. 2017). This behaviour is observed in all of the strong UV and optical broad emission lines and is characterised by a significant drop in emission line flux and an absence of response to continuum variations lasting $\approx 40$ days, bracketed by two shorter duration (10-15 days) "transition" periods which trace the departure from "normal" emission line behaviour into, and then out of, the anomaly. These transition periods are characterised by a reduced emission line flux and a weaker, though non-zero, response to continuum variations.

The appearance of the anomalous emission-line behaviour mid-way through the 2014 campaign is the first reported incidence of a breakdown in two of the fundamental assumptions that underpin broad emission-line reverberation mapping studies, specifically:

(1) There exists a simple causal relationship between the observed continuum and broad emission-line flux variations, and

(2) The observed continuum variations are a smoothed and scaled version (and are thus a suitable proxy) for the unobserved driving continuum variations at ionising energies.

This new and unexpected behaviour may provide new insights into the generation and transport of energy in the vicinity of the black hole.

\subsection{The anomalous behaviour as a diagnostic tool}

Goad et al. (2016) proposed two possible scenarios for the anomalous broad emission line behaviour: (i) temporary obscuration by a veil of material lying between the ionising 


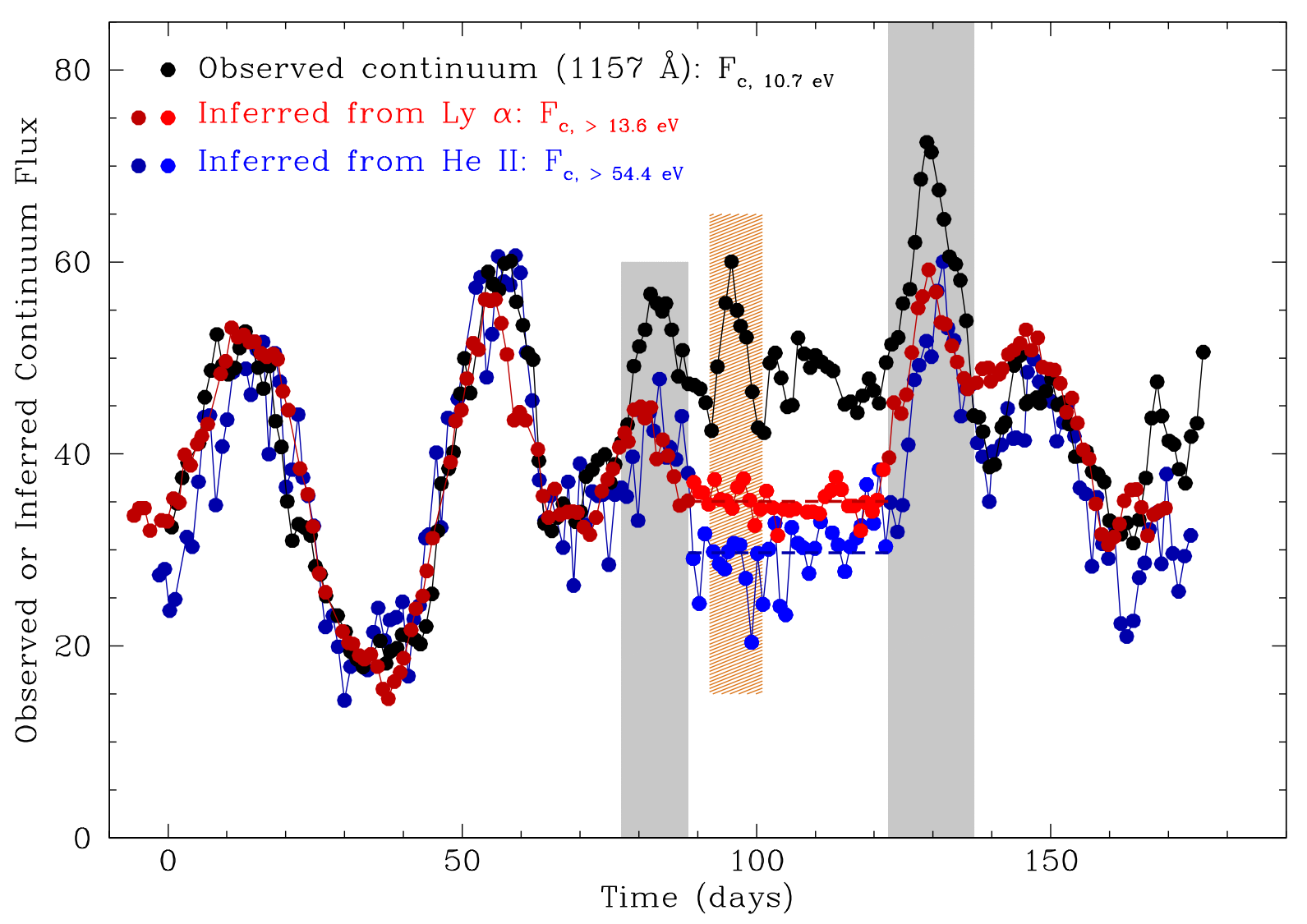

Figure 1. A comparison between the $H S T / \operatorname{COS} \lambda 1157 \AA$ continuum band (black points) and our inferred proxy continuum responsible for driving the Ly $\alpha$ (red points) and He II $\lambda 1640 \AA$ (blue points) emission-line variations. Grey shaded regions mark the time of approximate onset and exit of the anomaly. The orange shaded region, centred near day 95, indicates the most significant continuum event (at $\lambda 1157 \AA$ ) that occurred during the period of anomalous emission-line behaviour. The dashed horizontal lines indicate the inferred average values found during the anomalous period, colour-coded as above.

continuum source and the inner BLR that absorbs some fraction of the incident ionising continuum, or (ii) an intrinsic change in the strength, shape and variability behaviour of the ionising continuum. Since the anomaly is seen in all of the broad emission lines, though with differing flux deficits, any obscuring veil must be located at small BLR radii. The broad emission line flux deficits during the anomalous period are large, $\approx 25 \%$ for the higher ionisation lines, requiring a large solid angle for the obscuring material. However, while an obscuring veil could reproduce the observed drop in emission line strengths, it would not readily explain the absence of response of the broad emission lines to the modest, yet significant, UV-optical continuum variations that were present during the anomalous period, for example, the large continuum event starting at HJD 2,456,785 (day 94, refer to Figure 1 and §2.1). Consequently, Goad et al. (2016) favoured an intrinsic change in the strength and shape of the ionising continuum as the more likely explanation (see also Mathur et al. 2017). Whatever is the origin, it is evident that during the anomalous period much of the ionising continuum incident upon the BLR de-cohered in behaviour from the far- $U V$ continuum along our line of sight, both in average flux level and in variability (Goad et al. 2016). This is the subject of future work. Here, we pursue the use of the anomalous behaviour of the broad emission lines to illuminate two important aspects of the variable continuum.

First, Goad et al. (2016) used the observed broad emission line behaviour pre- and post- anomaly as a proxy for estimating the "expected" emission line behaviour based on the observed far-UV ( $\lambda 1157 \AA$ ) continuum variations as though the anomaly had not occurred. From this they were able to estimate the flux deficit in each of the broad emission lines during the anomalous period. Here we adopt an alternative approach, and use key diagnostic emission lines to trace the changes in the incident SED at ionising energies that must have occurred in order to produce the emission line behaviour that we observe. The importance of this difference in approach cannot be understated. Revealing the underlying behaviour of the ionising continuum is a key ingredient for recovering the BLR transfer function (e.g., Horne, Welsh, 
\& Peterson 1991; Welsh \& Horne 1991), and for constructing dynamical models of the BLR (Pancoast et al. 2012, 2014a,b; Barth et al. 2011, 2013) ${ }^{1}$, and is thus a necessary step toward achieving one of the principal goals of the AGN STORM campaign. Second, we search for the signature of the anomalous behaviour within the measured continuum bands to identify the presence of continuum light emanating from the BLR. These are described in turn in $\S 2$ and $\S 3$, below.

\section{INFERRING A PROXY FOR THE IONISING CONTINUUM LIGHT CURVE}

To infer a proxy for the ionising continuum's light curve, and in so doing track changes to its spectral energy distribution (hereafter, SED) incident upon the BLR, we analyse the light curves of two key diagnostic emission lines, Ly $\alpha$ and He II $\lambda 1640 \AA$. Ly $\alpha$, a resonance line and among the strongest UV emission lines, is sensitive to the number of ionising photons at energies above the Lyman limit $(\mathrm{E}>13.6 \mathrm{eV})$. He II $\lambda 1640 \AA$, on the other hand, is a high-excitation recombination line and is sensitive to the flux of photons with energies above $54.4 \mathrm{eV}$. He II, though far weaker than $\operatorname{Ly} \alpha$, has a small lag $(\approx 2.5-3$ days) relative to the shortest accessible UV continuum band $(\lambda 1157 \AA)$ and consequently is expected to suffer little geometric dilution ${ }^{2}$ in its response. Other than a small shift in delay, He II is therefore expected to faithfully track the ionising photon flux at higher energies.

Assuming that the $\lambda 1157 \AA$ continuum band faithfully tracks the continuum at ionising energies ${ }^{3}$, and that the emission-line variations arise from a narrow range in delays, we can infer a suitable proxy for the driving continuum as seen by the broad emission line region by simply shifting in time and scaling in amplitude, the observed emission line light curve to match the observed UV continuum variations outside of the anomalous period (see e.g., Goad et al. 2016, their Figure 1c). The required scale factor for each emissionline, after normalising to their mean values, is simply the

1 A key assumption underpinning this work is that the timevariable behaviour of the shortest wavelength continuum band at $\lambda 1157 \AA$ ( $\lambda 1138 \AA$ in the rest-frame of the source), is a suitable proxy for the continuum at ionising energies during times outside of the anomaly. While this band lies closest to the driving continuum and is the least contaminated by stellar light from the host galaxy, it is likely a temporally smoothed, reduced-in-amplitude, and slightly delayed version of the actual driving continuum.

2 Photoionisation model calculations and observations indicate that the broad emission-lines originate over a broad range in physical conditions and distances from the central source of ionising photons. That being the case, the observed emission-line light curve will in general be reduced in amplitude and delayed in time, depending on details of the mapping between source-cloud distance and the observer time-delay, as well as the characteristic time scale of the continuum variations relative to the relevant light travel times for a particular emission line (e.g., Goad \& Korista 2014). Together, these effects are referred to as geometric dilution of the emission line response.

3 The inferred driving continuum light-curve determined by Starkey et al. (2018) for this campaign, does not resemble the soft or hard band X-ray light-curves, but is instead a good proxy for the $1157 \AA$ light-curve, further justifying this assumption. inverse of the responsivity, the power-law index relating the observed continuum and broad emission line fluxes, as reported by Goad et al. (2016), their Table $2^{4}$. The smaller than expected delays and emission-line responsivities, and consequently large scale factors, likely indicate a significant contribution to the line emission from a large reservoir of gas which is largely unresponsive to the uncharacteristically short timescale (relative to previous campaigns) ionising continuum variations. That is, the BLR in NGC 5548 is much larger than would be inferred from a simple crosscorrelation analysis of the UV continuum and emission-line light curves. This conclusion is supported by results from reverberation mapping analyses of this source, which indicate that the BLR in NGC 5548 is spatially extended (Horne et al. 2019, AGN STORM paper IX, in prep). The recovered 1-d response functions $\Psi(\tau)$ span delays of 0 to 50 days, and though the bulk of the response is on short timescales, there is significant response for many lines, but particularly for $\mathrm{H} \beta$, on timescales as large as $\sim 40$ days. This is to be compared to a CCF centroid of $\sim 6$ days for $\mathrm{H} \beta$ relative to the $1157 \AA$ continuum band for the full campaign (Pei et al. 2017).

In Figure 1 we show the $H S T / C O S \lambda 1157 \AA$ UV continuum light curve (black points) together with the inferred proxy continua responsible for driving the observed emission-line variations in Ly $\alpha \lambda 1216$ (red points) and He II $\lambda 1640 \AA$ (blue points). Delays and scale factors for each line are here determined for days occurring before the onset of the anomaly (i.e., before HJD $2456,766.1094, \approx 75$ days after the start of the HST/COS campaign). Remarkably, all that is required to match well the observed continuum variations outside of the anomalous period is a simple scaling in amplitude and temporal shift of the Ly $\alpha$ and He II broad emission line light curves. We find little evidence for significant temporal smearing of the broad emission-line light curves outside of the anomalous period (compare the C IV emissionline light curve to the one inferred directly from the $\lambda 1157 \AA$ continuum, Goad et al. 2016, their Figures 1c,d). This suggests that the variable part of each emission-line arises from a surprisingly narrow range in delays $(<10$ days) relative to the reported lag values, i.e., the response functions must be relatively narrow in time delay.

Figure 1 next illustrates that the most striking difference between the observed $\lambda 1157 \AA$ continuum light curve and the continuum that drives the broad emission lines, as inferred from the behaviour of the Ly $\alpha$ and He II emissionlines, is the large deficit in continuum flux: $\approx 30 \%$ for Ly $\alpha$, $\approx 40 \%$ for $\mathrm{He} \mathrm{II}$, and $\approx 20 \%$ for $\mathrm{H} \beta$ (not shown) during the time of the anomaly. In Figure 2 we show the ratio of the inferred proxy driving continua for $\operatorname{Ly} \alpha$ (red points) and He II (blue points) relative to the observed $\lambda 1157 \AA$ continuum band. This indicates a significantly larger reduction in the continuum flux responsible for driving variations in the broad He II emission line relative to the continuum band at $\lambda 1157 \AA$ than there is for that driving $\operatorname{Ly} \alpha$. Thus during the anomaly the ionising continuum as incident upon the

4 Alternatively, the continuum and emission line light curves can be scaled to one another using the ratio of their root mean square variations. These are mathematically equivalent for small variations about some average value (Krolik et al. 1991). 


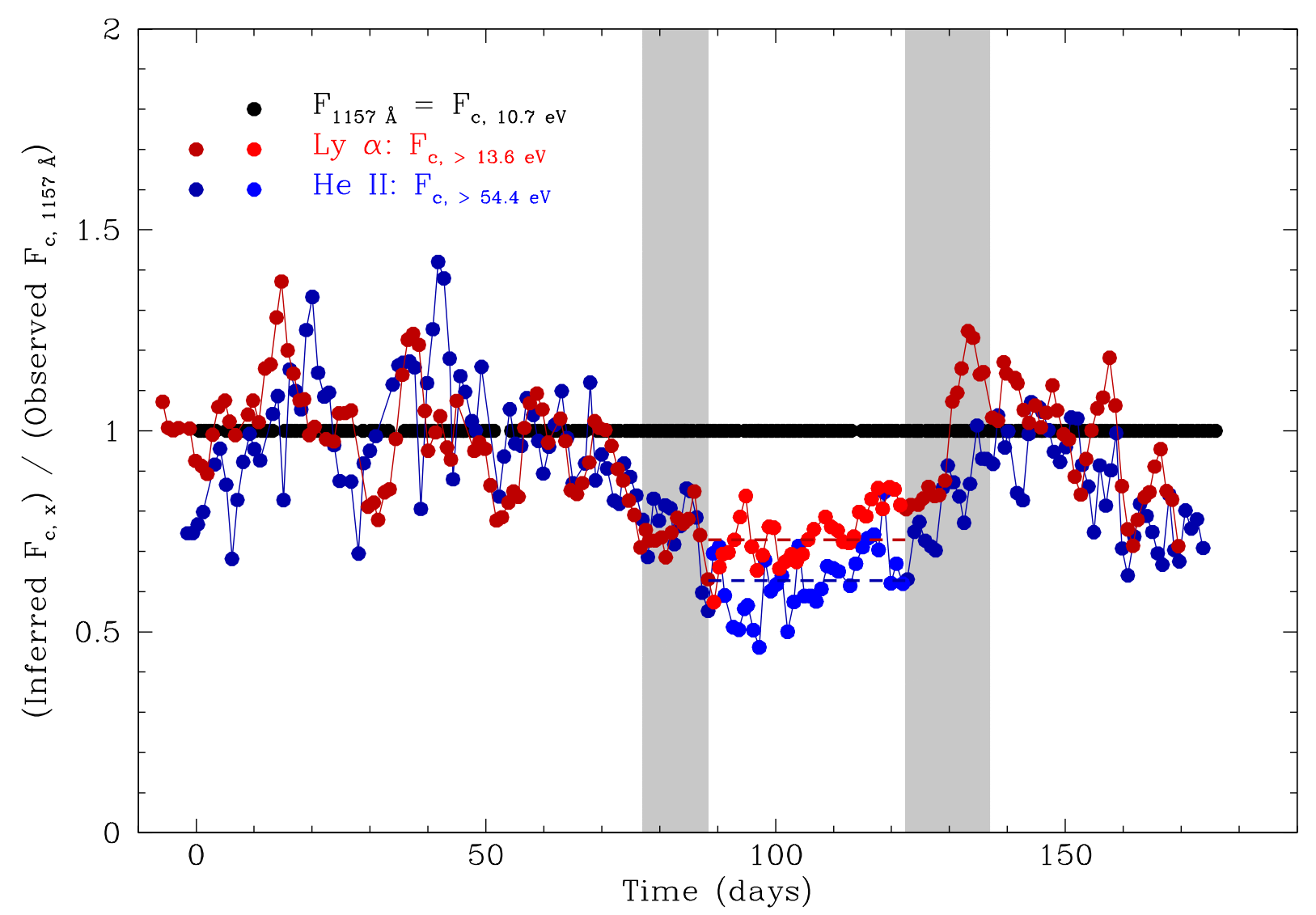

Figure 2. The ratio of the inferred proxy driving continuum bands for Ly $\alpha$ and He II $\lambda 1640 \AA$ relative to the observed continuum at $\lambda 1157 \AA$. The inferred He II continuum shows a larger decline relative to $\lambda 1157 \AA$, than the inferred Ly $\alpha$ continuum. The dashed horizontal lines indicate the inferred average values for this ratio found during the anomalous period, colour-coded as above.

BLR became weaker and softer relative to the continuum at $\lambda 1157 \AA$. We note here that this analysis is based on integrated broad line fluxes in Ly $\alpha$ and He II. The actual reductions in the 1 and 4 Rydberg continua photon fluxes incident upon the BLR are therefore likely somewhat larger than the values quoted above, which are geometrically diluted by effects associated with light travel time. As a matter of fact, this same analysis technique, when applied to individual radial velocity bins, finds larger flux deficits in the line wings of $\mathrm{C}$ IV than in the line core, consistent with this inference. We will explore these aspects in future work.

Finally, also illustrated in Figure 1 are three shaded regions, two grey shaded regions which identify local peaks in the $\lambda 1157 \AA$ continuum at the beginning and end of the anomaly, and an orange shaded region marking the largest $\lambda 1157 \AA$ continuum event that was observed during the anomalous period. The grey shaded regions can be identified with the two transition periods reported by Goad et al. (2016) marking the transitions in to and out of the anomaly. During the transition intervals, the inferred Ly $\alpha$ and He II ionising continuum flux track the continuum at $\lambda 1157 \AA$, but with a reduced flux and smaller variability amplitude. Impor- tantly, there is no equivalent feature in the inferred proxy continua (i.e., from $\mathrm{Ly} \alpha$ or He II) to the large observed continuum event in the $\lambda 1157 \AA$ continuum band indicated by the orange shaded region. As noted by Goad et al. (2016), and given the apparent small spread in delays for the broad emission lines referred to previously, we should have been able to measure significant variations in the broad emissionlines related to this feature, and yet we do not. Why the ionising continuum no longer tracks the $\lambda 1157 \AA$ continuum during the anomalous period is unclear, but presumably is related to the redistribution of photons in energy.

In the following section, we utilise both of the above characteristic differences (shown in Figure 1) pointing to a decohering of the bulk of the driving ionising continuum incident upon the BLR from the observed far-UV continuum during the anomalous period, to identify anomalous behaviour in the UV-optical continuum band measurements. 


\section{ANOMALOUS BEHAVIOUR IN THE UV-OPTICAL CONTINUUM BANDS}

Thus far, the anomaly has been discussed in terms of its effect upon the observed broad emission-line flux light curves. Whether the anomaly is present within the UV and optical continuum bands has not been explored. However, a feature of photoionisation model predictions of dense BLR gas is a significant diffuse continuum, in addition to broad emission-lines (KG01; Lawther et al. 2018). For example, KG01, constructed a local optimally emitting cloud model (hereafter LOC, Baldwin et al. 1995) for the broad emission lines in NGC 5548 designed to match the observed luminosities of the two strongest UV emission lines, Ly $\alpha$ and C IV (Clavel et al. 1991; Krolik et al. 1991; Korista et al. 1995). They found that their model BLR produces (in addition to emission lines) a significant thermal diffuse continuum component spanning the entire $U V$-optical-near $I R$ continuum. This diffuse continuum component is largely dominated by the $\mathrm{H}-\mathrm{He}$ recombination (free-bound) continuum, with smaller contributions from free-free emission as well as scattering from free electrons and neutral hydrogen (see Korista \& Ferland 1998). Since the diffuse continuum originates in the spatially extended BLR, it will display a broad range in variability amplitude and delay. While the lag spectra for both the disk and the DC components generally increase to longer wavelengths, their detailed shapes differ. The accretion disk lags are expected to go as $\tau \propto \lambda^{4 / 3}$ (Cackett et al. 2007), the DC has more of a sawtooth shape with peaks e.g., near the Balmer and Paschen breaks (KG01; Lawther et al. 2018). While the differences are better delineated in data with much higher spectral resolution (e.g. NGC 4593; Cackett et al. 2018), it is much harder to resolve with broadband photometry such as used here.

For conditions appropriate to the BLR, a significant diffuse continuum component is largely unavoidable. Yet, interpretation of the larger than expected continuum inter-band delays have instead preferred to appeal to alternate models for describing the structure of the disk, including: truncated disks (Narayan 1996), truncated disks with an additional reprocessing region (Gardner \& Done 2017), inhomogeneous disks (Dexter \& Agol 2011), patchy disks (Starkey et al. 2017), disk atmospheres (Hall et al. 2018), and tearing disks (Nealon et al. 2015). The reduced variability and larger than expected delays measured at all wavelengths, and in particular in the vicinity of the Balmer continuum, relative to that predicted by the standard disk model (Edelson et al. 2015, 2017, 2018; Fausnaugh et al. 2016; Starkey et al. 2017, McHardy et al. 2018) we argue is broadly consistent with what one might expect if the UV-optical continuum bands are significantly contaminated by a diffuse continuum emitting from the same gas that emits the broad emission lines (KG01; Lawther et al. 2018).

In general, the longer wavelength continuum bands will be reduced in amplitude and smeared in time relative to the shorter wavelength continuum bands because:

- the underlying UV-near-IR continuum arises from thermalised emission of an accretion disk (or some other accretion geometry) with a spatial gradient in gas temperature, such that $\mathrm{kT}$ diminishes with increasing radius,

- whatever the mechanism behind the variations in lumi- nous flux from the disk, its transfer function is not a deltafunction,

- there may be a significant DC contribution from the BLR.

All of the above may be contributing to the observed continuum variations at some level, and all will act to reduce the continuum inter-band correlations.

Here we take advantage of the presence of the anomaly to identify the presence of the diffuse continuum component. If the diffuse continuum component is important, we should see the anomaly's effect in the observed continuum bands, and should follow a pattern of behaviour that is similar to that seen in the emission lines.

In particular, we predict that the diffuse continuum would have undergone a significant drop in flux during the anomaly in contrast to the $\lambda 1157 \AA$ continuum band ${ }^{5}$. Additionally, we predict that the most significant continuum event observed during the anomalous period (Figure 1, orange shaded region) will be significantly weaker in the longer wavelength DC band light-curves, as it is for the integrated flux in broad emission lines. Because the behaviour of the DC component is controlled by the same driving continuum inferred for the broad emission-lines, then any significant presence of the DC will act to suppress the inter-band continuum correlations during the anomalous period relative to their behaviour at other times.

To test this expectation we here investigate the relationship between the continuum at longer wavelengths relative to the shortest accessible UV band $\lambda 1157 \AA$, a band for which photoionisation model calculations suggest has only a weak contribution from the BLR diffuse continuum (KG01), and which is also least likely to have contributions from the wings of broad emission lines, when compared to other continuum bands (Kriss et al. 2019). Specifically, we test for differential behaviour between this band and the longer wavelength continuum bands for time periods spanning the anomaly and otherwise.

The method we employ is illustrated in Figure 3. First, we shift each of the longer wavelength continuum bands backwards in time according to their measured delay with respect to the $\lambda 1157 \AA$ continuum band. We use the delays reported by Fausnaugh et al. (2016), and Pei et al. (2017), determined using the software package JAVELIN in order to shift the light curves. This is mathematically equivalent to assuming the signal is reprocessed in a face-on ring. The $\lambda 1157 \AA$ continuum band and its longer wavelength pair are then interpolated onto a common grid (using simple linear interpolation). Only points which are close in time $(<0.5$ day difference) and common to both light curves are plotted (grey points). This avoids extrapolation beyond the ends of the light curves and ensures that the number of useful pairs of points is preserved.

Referring to the lower panel of Figure 3, for each light curve pair we highlight in red points taken from the middle of the anomalous period (days $86-123$ ). The blue points in

5 Photoionisation models indicate that the DC contribution to the $1157 \AA$ continuum band is small in comparison to most longer wavelength UV-optical continuum bands. However, we can not exclude the possibility that the $1157 \AA$ continuum band itself exhibits characteristics of anomalous behaviour of the DC. 


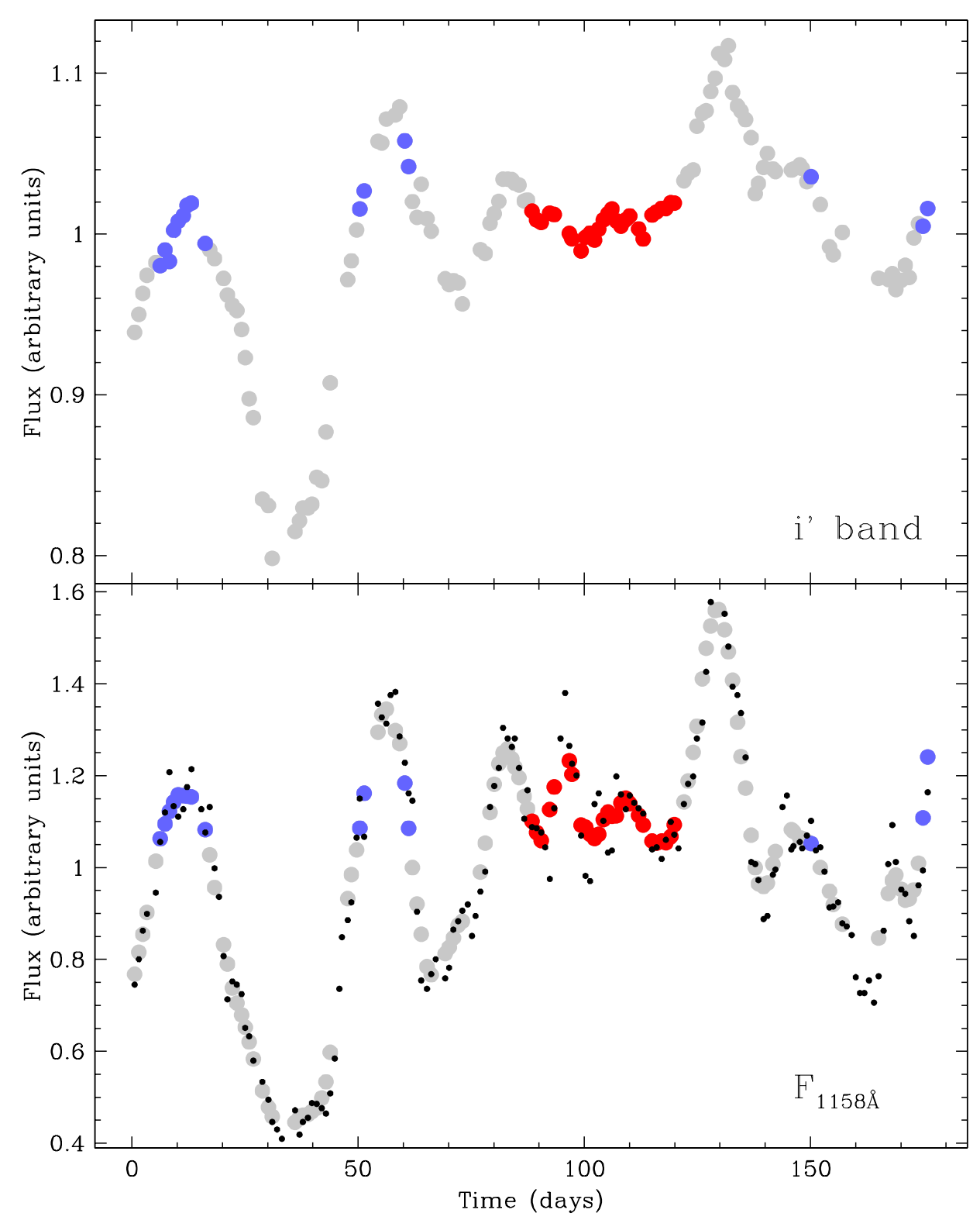

Figure 3. A comparison between the delay-shifted ground-based $i^{\prime}$ continuum light curve (grey points, upper panel), the original, unsmoothed $\lambda 1157 \AA H S T / C O S$ UV continuum (black points, lower panel), and the $\lambda 1157 \AA$ continuum after convolving with the disk transfer function (grey points, lower panel, see text for details). Each light curve has been normalised to its mean value. In the lower panel, red and blue points indicate portions of the light curve covering similar ranges in flux within (red) and outside of (blue) the anomaly. In the upper panel red and blue points indicate the corresponding $i^{\prime}$-band points over the same time intervals.

the bottom panel indicate those days outside of the anomaly for which the $\lambda 1157 \AA$ continuum band shows approximately the same range in flux as found during the anomalous period excluding the transition regions (indicated in red). This allows us to compare the strength of the flux-flux correla- tions inside and outside of the anomalous period over the same range in flux level as that within the $1157 \AA$ continuum band. Nonetheless, it is the case that the amplitude and timescale for the variability are smaller on average inside of the anomaly than outside of the anomaly (e.g., Sun 
et al. 2018). Since we cannot control for this latter effect, and because a reduced correlation is a natural consequence of reduced variability amplitude in the presence of noise, the test described here is by no means definitive, and hence our conclusions are not without bias. We investigate this further in $\S 3.2$.

In essence the blue points indicate what the "normal" behaviour of the diffuse continuum from the BLR should be for the range in $\lambda 1157 \AA$ continuum flux present during the anomalous period, modulo the bias discussed above. The top panel of Figure 3 identifies the same blue and red points in time for the $i^{\prime}$ band. Light curve pairs in the other continuum bands are then similarly transposed onto flux-flux diagrams with the same colour coding (refer to Figures 4 and 5).

Immediately apparent from Figures $4-5$, is that even though the blue points are spliced together from five essentially randomly-selected segments, for the majority of continuum bands, they are more tightly correlated with each other than the contiguous red (anomalous period) points. That is, over the observed range in $\lambda 1157 \AA$ continuum flux present during the anomalous period, the longer wavelength inter-band continuum variations correlate better outside of, rather than inside of the anomalous period. We suggest that the UV (perhaps also including $\lambda 1157 \AA$ ) and optical continuum bands therefore must also be affected during the anomaly, and in a similar fashion to the emission lines.

To quantify the extent to which the longer wavelength continuum bands decorrelate from the $\lambda 1157 \AA$ continuum band during the anomalous period we perform a simple Spearman rank correlation between the flux-flux pairs for the red and blue points. These are indicated in Figure 5, alongside zoomed in versions of the flux-flux correlations ${ }^{6}$. The measured correlation coefficients $\rho$ and p-values (in brackets) are indicated in Figure 5 and summarised in Table 1 (columns 4 , and 5 ). The correlation coefficients for the blue points are in the majority of cases larger than those for the red points, indicating that the variations in the longer wavelength continuum bands are indeed more weakly correlated with the $\lambda 1157 \AA$ continuum band during the anomalous period. Since the red and blue points identified in individual light-curves are drawn from the same range in flux level and are thus of similar signal-to-noise, the weaker correlation found between flux-flux pairs taken from inside of the anomaly must arise due to intrinsic differences in lightcurve behaviour.

While a reduced correlation between the short and longer wavelength bands arises naturally from a reverberating disk, this cannot explain the difference in behaviour inside and outside of the anomalous period. Significant contributions from a diffuse continuum emanating from BLR clouds would also act to further reduce the inter-band correlations. However, the observed differential behaviour, i.e., the reduced inter-band correlations found during the anomaly (red points) relative to those found outside of the anomaly (blue points) we suggest arises because the continuum bands contain a substantial amount of light from the

\footnotetext{
6 Spearman rank correlation coefficients are invariant to transformations of the form $y \rightarrow y-a$ or $y \rightarrow y / a$ and are thus insensitive to the presence of non-variable constant components (e.g., the host galaxy contribution).
}

BLR clouds, which as for the emission-lines, underwent a drop in flux and did not see the significant continuum event noted in Figure 1, centred on day 95 (orange shaded region).

Finally, we note that the contribution of non-variable sources (e.g., the host galaxy contribution, and reprocessed continuum from narrow line region gas) to the flux in the continuum bands is also likely wavelength dependent. However, our simple ranking scheme for measuring the correlation strength between the continuum bands is insensitive to these non-variable components.

\subsection{Disk transfer functions}

Shifting the longer wavelength continuum light curves in time, using a one-number estimate for the "size" of the continuum emitting region (relative to that emitting $\lambda 1157 \AA$ ) is a rather simplistic approach. In the standard disk model (Shakura \& Sunyaev 1973) each continuum band arises from a range in disk radii such that the longer wavelength continuum bands are not only delayed relative to those at shorter wavelengths, but their variations are also smaller in amplitude and smoothed in time, reducing the correlation between the bands (Collier 2001; Cackett et al. 2007, 2018; Edelson et al. 2015; Fausnaugh et al. 2016; Starkey et al. 2017). We test the significance of this "reduced correlation" by convolving the $\lambda 1157 \AA$ continuum light-curve with a model disk transfer function representing the response of a thin ring of material, with a size that corresponds to the measured lag between the longer wavelength continuum band and $\lambda 1157 \AA$ and viewed at and angle of 36 degrees relative to our line of sight to the disk. This inclination is equal to the best-fit value found for the disk orientation in NGC 5548 by Starkey et al. (2017), $36 \pm 10$ degrees, when fitting for the disk radial temperature profile, inclination $i$ and normalisation, similar to the inclination angle derived for the broad emission-line region in NGC 5548, 38.8 $8_{-11.4}^{+12.1}$ degrees (Pancoast et al. 2014), and in broad agreement with the inclination required to recover the virial factor $f$ for this source (Goad et al. 2012), and the inclination inferred for this source from the recovered broad emission-line velocity delay maps (Horne et al. 2019, AGN STORM paper IX, in prep.). This smoothed version of the $\lambda 1157 \AA$ continuum light-curve is then correlated against the longer wavelength continuum band following the procedure outlined above.

The results of this process are shown in Figures 6-7, and summarised in Table 1 (columns 6 and 7). Convolving with a disk transfer function acts to tighten the relationship between the $\lambda 1157 \AA$ and longer wavelength continuum bands. We also refer the reader back to Figure 3 for a direct comparison between the $\lambda 1157 \AA$ continuum light curve and that of one at longer wavelength. However, while the correlation coefficients are slightly modified, our main result still stands. Red points taken during the anomalous period are consistently less correlated with the $\lambda 1157 \AA$ continuum band than are the blue points. We note that Sun et. al. 2018 report significant differences between the delay and response amplitude between the Swift X-ray and far UV HST band $(\lambda 1367 \AA)$, indicating that the delays between these two bands are longer and more tightly correlated (coherent) in the normal state (outside of the anomaly) than in the anomalous state, further justifying our claim. 

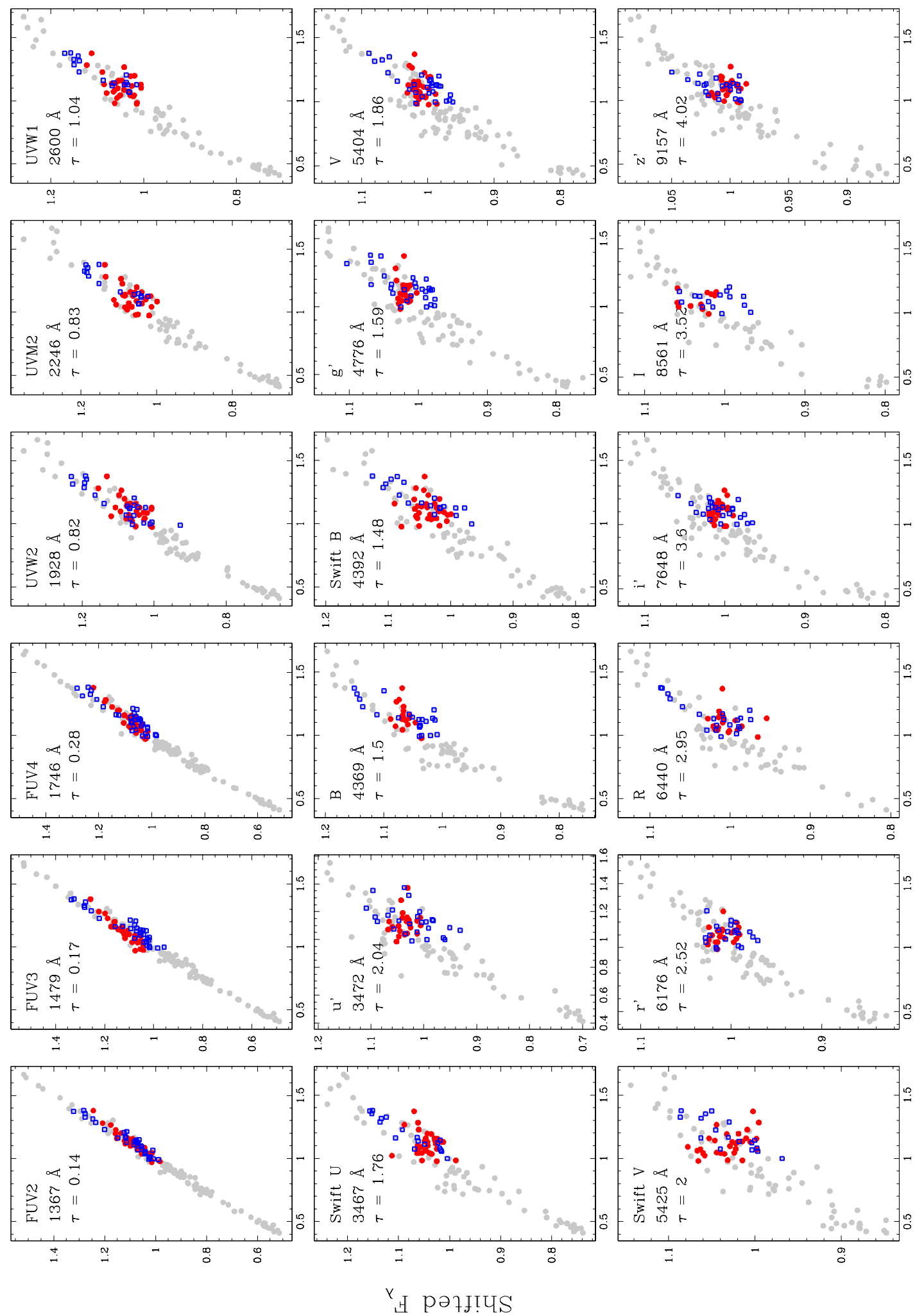

응

을

E

司

ق

要要目

营总

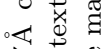

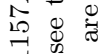

in

요읠 융

도웜

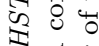

吾点

훔 용

胥

苟要

密

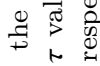

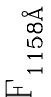

$\circ$ 뭉

究

응

要

空

ㅋ.

蛋.

政

范茟

设

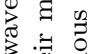

$3 . \overline{0}$

응

通

क्षित

धै

惫苟

壱

离量

मi

离部 

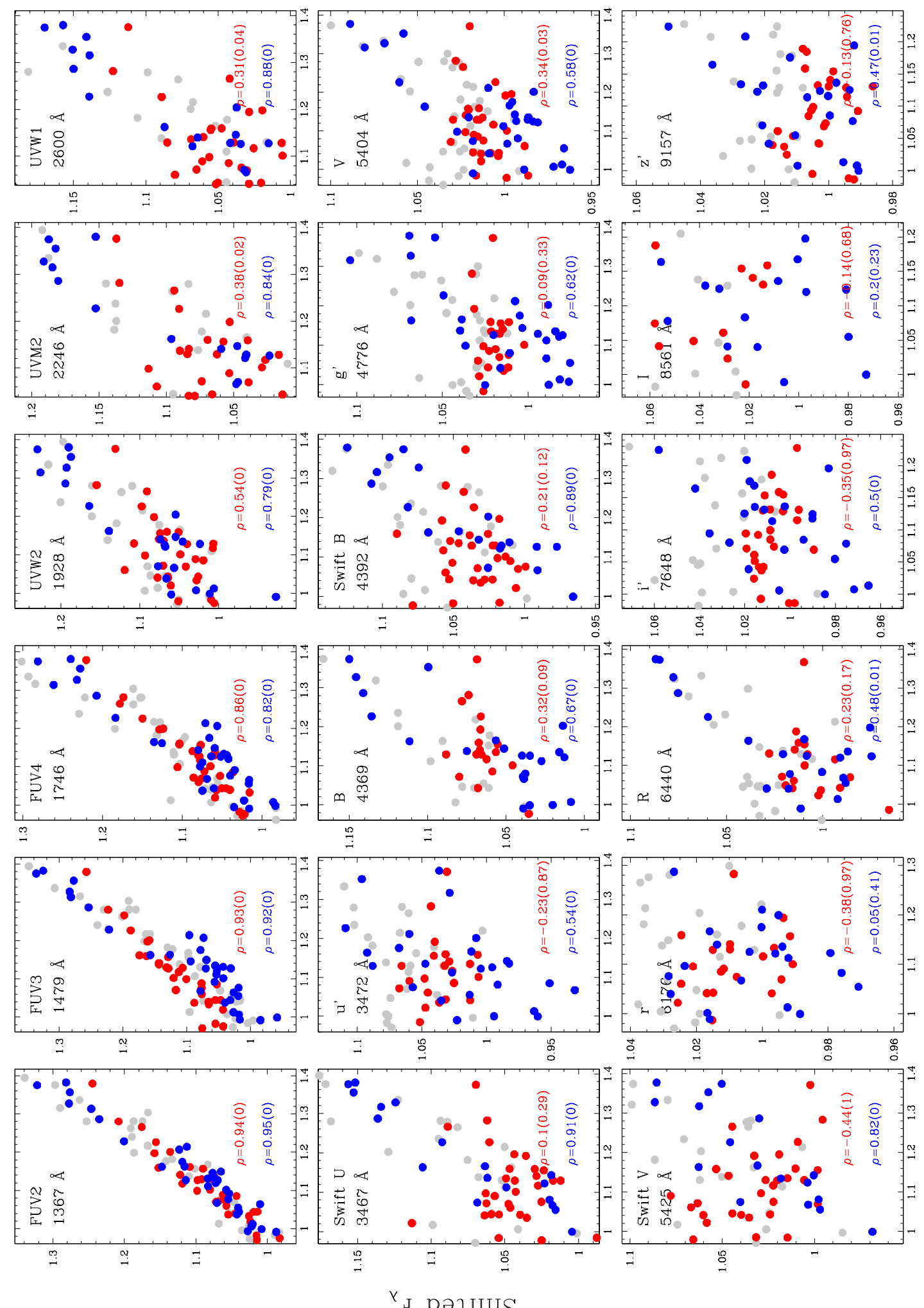

火出 рә7л!ЧS 

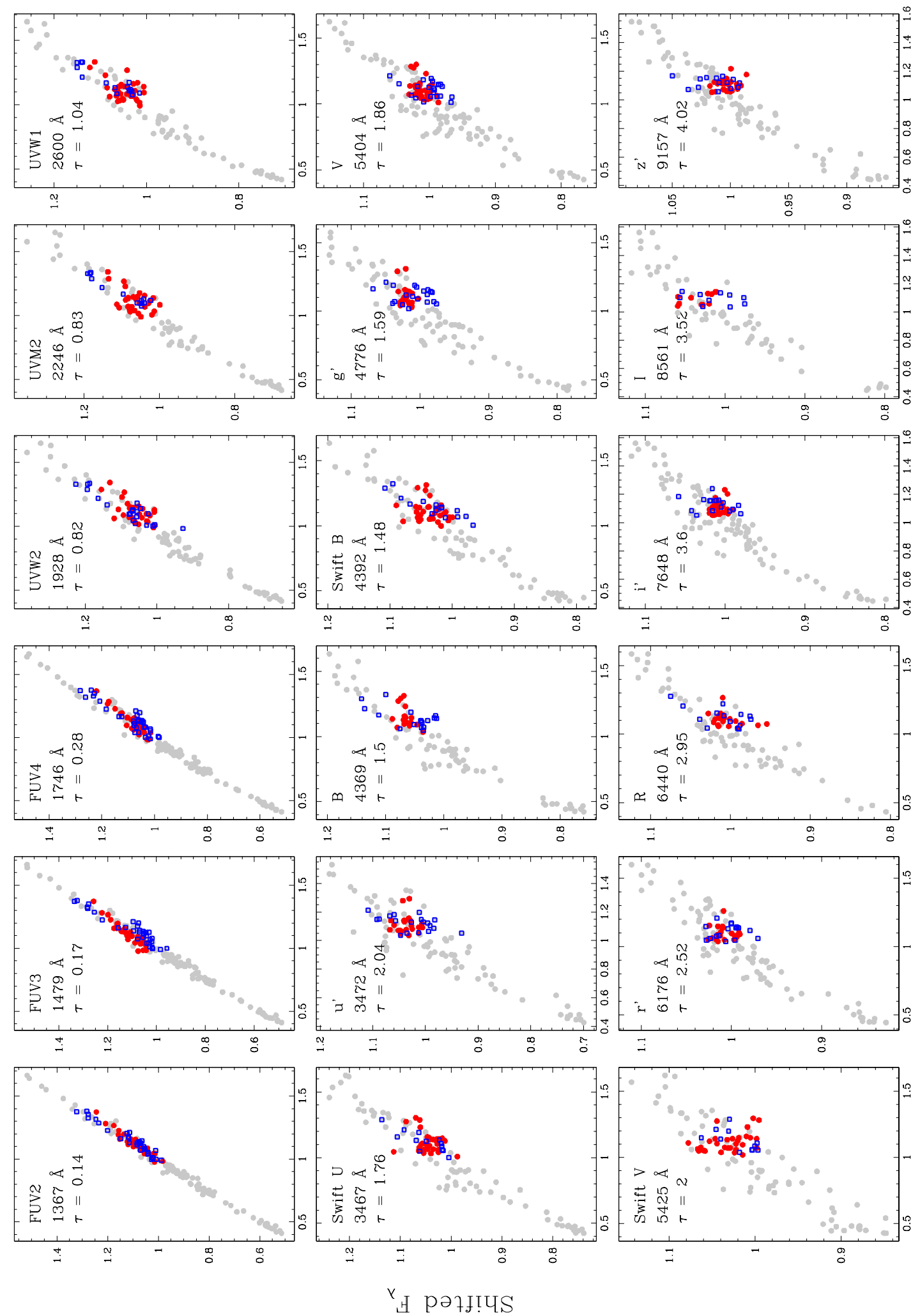

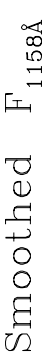



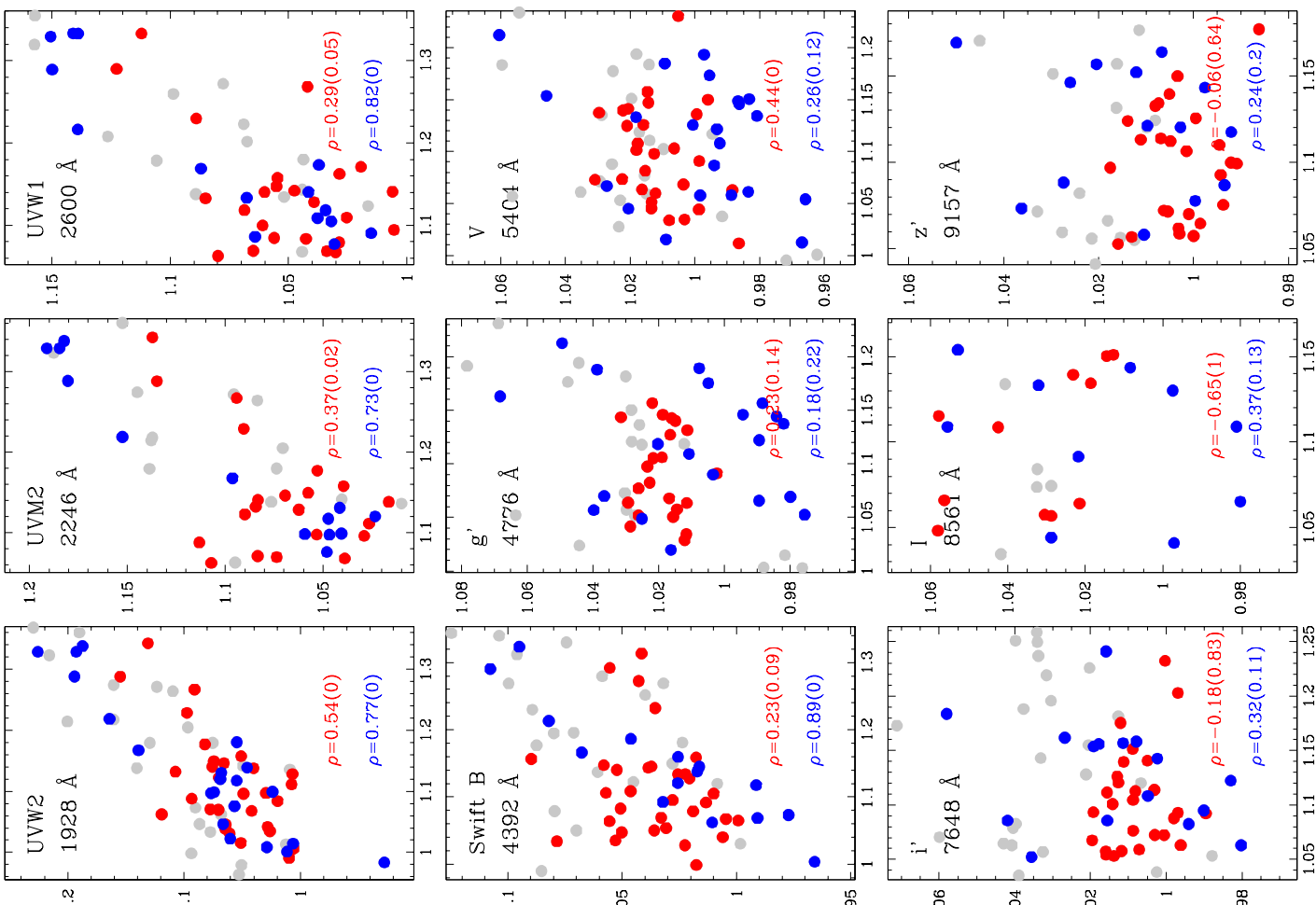

र

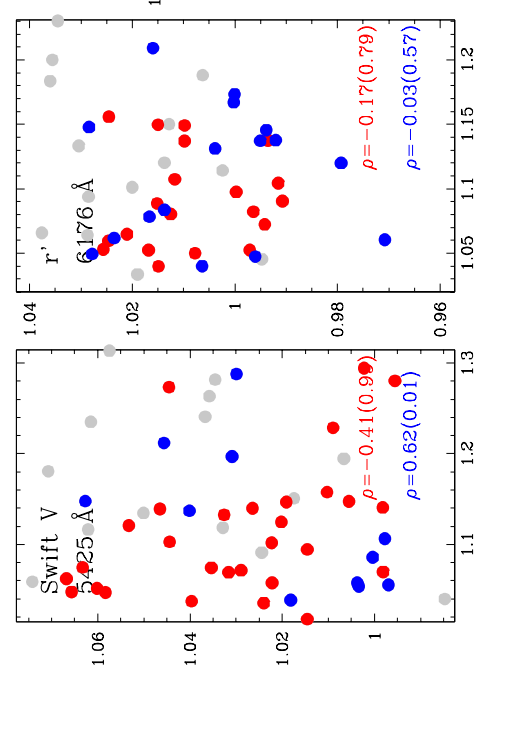


Table 1. Mean delays and Spearman rank correlation coefficients for the measured UV-optical-near IR continuum bands

\begin{tabular}{lrrrrrr}
\hline \hline Bandpass & $\begin{array}{r}\text { pivot } \lambda \\
(\AA)\end{array}$ & $\begin{array}{r}\text { delay }^{\dagger} \\
\text { (days) }\end{array}$ & $\begin{array}{r}\rho \\
(\text { red })\end{array}$ & $\begin{array}{r}\rho \\
(\text { blue })\end{array}$ & $\begin{array}{r}\rho(\mathrm{sm})^{\ddagger} \\
(\mathrm{red})\end{array}$ & $\begin{array}{r}\rho(\mathrm{sm}) \\
(\mathrm{blue})\end{array}$ \\
\hline HST/COS & 1367 & 0.14 & 0.94 & 0.95 & 0.94 & 0.96 \\
HST/COS & 1479 & 0.17 & 0.93 & 0.92 & 0.94 & 0.93 \\
HST/COS & 1746 & 0.28 & 0.86 & 0.82 & 0.87 & 0.82 \\
Swift UVW2 & 1928 & 0.82 & 0.54 & 0.79 & 0.54 & 0.77 \\
Swift UVM2 & 2246 & 0.83 & 0.38 & 0.84 & 0.37 & 0.73 \\
Swift UVW1 & 2600 & 1.04 & 0.31 & 0.88 & 0.29 & 0.82 \\
Swift U & 3467 & 1.76 & 0.10 & 0.91 & 0.12 & 0.83 \\
$\mathrm{u}^{\prime}$ & 3472 & 2.04 & -0.23 & 0.54 & -0.06 & 0.51 \\
B & 4369 & 1.50 & 0.32 & 0.67 & 0.38 & 0.39 \\
Swift B & 4392 & 1.48 & 0.21 & 0.89 & 0.23 & 0.89 \\
$\mathrm{~g}^{\prime}$ & 4776 & 1.59 & 0.09 & 0.62 & 0.23 & 0.18 \\
V & 5404 & 1.86 & 0.34 & 0.58 & 0.44 & 0.28 \\
Swift V & 5425 & 2.00 & -0.44 & 0.82 & -0.41 & 0.62 \\
$\mathrm{r}^{\prime}$ & 6176 & 2.52 & -0.38 & 0.05 & -0.17 & -0.03 \\
$\mathrm{R}$ & 6440 & 2.95 & 0.23 & 0.48 & 0.37 & 0.39 \\
$\mathrm{i}^{\prime}$ & 7468 & 3.60 & -0.35 & 0.50 & -0.18 & 0.32 \\
$\mathrm{I}$ & 8561 & 3.52 & -0.14 & 0.20 & -0.65 & 0.37 \\
$\mathrm{z}^{\prime}$ & 9157 & 4.02 & -0.13 & 0.47 & -0.06 & 0.24 \\
\hline
\end{tabular}

${ }^{\dagger}$ Measured delays are from Fausnaugh et al. (2016), and Pei et al. (2017) and are measured relative to the HST/COS $\lambda 1157 \AA$

continuum band.

$\ddagger(\mathrm{sm})$ indicates that the $\lambda 1157 \AA$ light-curve has first been convolved with a disk transfer function (see text for details).

\subsection{Additional tests}

We perform one additional test, assuming that the longer wavelength continuum light-curves are scaled, smoothed and delayed versions of the continuum bands at shorter wavelengths. We use the shortest wavelength continuum band from $H S T(\lambda 1157 \AA)$ as a reference, as photoionisation model calculations suggest that this band is likely least contaminated by diffuse continuum emission from BLR gas (KG01; Lawther et al. 2018). We then fix the delay of the longer wavelength bands relative to this reference band using values reported in Fausnaugh et al. (2016), and then scale the $H S T$ band according to the relation

\section{$\log F_{\lambda}$ (long) $=\alpha \log F_{\lambda}$ (short) $+K$.}

The HST band is then smoothed using a Gaussian smoothing kernel normalised to unit area. We then fit the smoothed, scaled version of the reference $H S T$ continuum band to the delay-corrected longer wavelength continuum band over those epochs which lie outside of the anomaly identified by Goad et al. 2014, and indicated in Figure 8 by the vertical blue dashed lines (corresponding to epochs JD2456766.1-JD2456829.8). That is, we implicitly assume that the out of anomaly data indicate what the normal response of a continuum band to a given amplitude driving continuum event should look like. The fit is then optimised using $\chi^{2}$ minimisation, comparing (as before) only those pairs of points in both light-curves which lie within \pm 0.5 days of one another, i.e., no where do we interpolate the light-curves, as for sparsely sampled light-curves, such an approach gives too much weight to interpolated points in determining the fit. We optimise the parameters - scale factor $\alpha$, offset $K$ and full-width at half-maximum (FWHM) of the smoothing kernel, by minimising the residuals between the two light curves in the fitted region. The results of our fitting procedure are illustrated in Figure 8, which shows a fit to the ground-based $i^{\prime}$ light-curve, among the best-sampled ground-based light-curves (under-sampled light-curves bias the fitting procedure to find smoother fits). Figure 8 shows the delay-corrected longer wavelength continuum band (red points) together with the scaled, and smoothed shortest wavelength $H S T$ band $(\lambda 1157 \AA$, black points).

Averaging the fluxes of near-contemporaneous (within \pm 0.5 days) data pairs taken from the centre of the anomalous period (JD2456777.43 - JD2456814.97), indicates that in general the smoothed and scaled HST light-curve overpredicts the flux in the longer wavelength continuum bands during this time period. This effect, though small, is most apparent in the longer wavelength optical bands $u^{\prime}, g^{\prime}, r^{\prime}, i^{\prime}$ and $z^{\prime}$, i.e., the same wavelengths at which the diffuse continuum contribution to the measured continuum flux (and so delay signal) is predicted to be strong. It is also seen to a lesser extent, in the Swift UVW1, UVW2 and U bands, where the smoothed and scaled HST light-curve lies above the majority of the red points.

In particular, the continuum event centred on day 95 (epoch JD2456785.7), highlighted in orange in Fig 1, if present in the driving continuum light curve, is of sufficient amplitude and duration to drive an observable response in the more slowly varying broad emission-lines (Goad et al. 2016). As we show later, it is also more than capable of driving a similarly large amplitude response in the longer wavelength continuum bands (and which vary on even shorter timescales). However, this feature is not seen either in the integrated broad emission-line light-curves reported by Goad et al. (2016), nor in the longer wavelength continuum lightcurves presented here (e.g., compare the black points and/or blue solid line with the red points in Figure 8). Instead, the 
longer wavelength continuum bands display reduced amplitude variability relative to that predicted, and a reduced flux, during the anomalous period. While part of this effect may be attributed to contamination of the Swift UV/optical and ground-based optical broad band filters by broad emissionlines, the longer wavelength $i^{\prime}, I$ and $z^{\prime}$ continuum bands are free of strong emission lines. ${ }^{7}$ The degree to which the diffuse continuum from the BLR affects the continuum band light curves will depend on its contribution to the measured continuum band flux. We explore this in future work.

We repeat this analysis fitting instead to the in-anomaly points only. While this reduces the flux deficit between the smoothed HST light-curve and longer wavelength continuum bands (as expected) during the period of the anomaly, the peak centred at day 95, and apparent in the smoothed $H S T$ light-curve, is not present in the longer wavelength continuum bands. Removal of this peak requires a degree of smoothing not warranted by the data, and as a consequence results in a poor fit to the out-of anomaly data (due to the smaller amplitude variability of the in-anomaly data). By expanding the number of fit parameters to include a linear background component (solid blue-line), we exclude the possibility that the flux deficit in the anomalous region arises due to a tilt in the light-curves relative to one another. For completeness, we have also performed fits optimised to the pre-anomaly data only and to the post-anomaly data only (where possible). In all cases, the excess flux in the region of the anomaly predicted by the model remains.

All of these experiments support the notion that there is a flux deficit in the longer wavelength continuum bands, which generally should have larger contributions from the BLR diffuse continuum, relative to that at $\lambda 1157 \AA$, and that there exist significant differences in the response amplitude of the continuum light-curves between the start and end of the campaign.

\section{DISCUSSION}

We have used the observed variations of key diagnostic emission lines to show that the anomalous behaviour observed among the broad emission lines in NGC 5548 (Goad et al. 2016; Pei et al. 2017) most likely arises from a change in the strength and shape of the ionising continuum incident upon the BLR gas. Specifically, the higher energy continuum band responsible for driving variations in He II $\lambda 1640 \AA$ (at energies above $54.4 \mathrm{eV}$ ) shows a larger decline relative to the $\lambda 1157 \AA$ continuum band than the continuum just shortward of the Lyman limit and responsible for driving variations in Ly $\alpha$. The ionising SED incident upon BLR clouds is both weaker and softer than expected relative to the $\lambda 1157 \AA$ continuum band during the anomalous period. In the context of

\footnotetext{
7 Though thermal emission from dust may contribute to the continuum flux at longer wavelengths, the size of the dusty torus in NGC 5548 is large ( $\approx 50$ light-days; Suganuma et al. 2006) even when compared to the typical formation radius of major emissionlines. Coupled with the relatively small amplitude and uncharacteristically short continuum variability timescales present during the AGN STORM campaign, it is unlikely that thermal dust emission contributes significantly to the observed continuum variations at longer wavelengths.
}

a scenario in which intrinsic changes in the SED took place, a steepening in the EUV power-law index by $\approx 0.3$ would approximately reproduce the observed unexpected decreases in the emission-line equivalent widths during the anomalous period (Korista, Baldwin, \& Ferland 1998; see their Figure $1 b)$.

Supporting evidence for a change in the ionising SED at EUV energies can be found in simultaneous Swift/XRT and Chandra X-ray spectra of NGC 5548 (Mathur et al. 2017). In particular, the higher $\mathrm{S} / \mathrm{N}$ Swift observations indicate a dramatic increase in the soft X-ray flux (soft excess) at energies of $0.3-2 \mathrm{keV}$, starting $\approx 10$ days prior to the onset of the anomaly, as determined from the UV broad emission lines (see Figure 1). This soft excess reaches a maximum in flux near the time of the transition into the anomalous period (approximately near day 74), and then declines thereafter to its pre-anomaly value some $\approx 40$ days later. While the causal nature between the observed soft X-ray flare and the anomaly is not yet clear, we believe a link between the two phenomena to be highly probable. We also note that the observed soft X-ray emission is along the line of sight, and our viewpoint may not be representative of that seen by the BLR, i.e., there are likely many different sight lines between the continuum source and BLR clouds which do not intercept our line of sight. Anomalous behaviour in the intrinsic narrow absorption lines, and in particular those of high ionization, in NGC 5548 add further supporting evidence for a change in the SED at soft X-ray energies along our line of sight (e.g., Kriss et al. 2019, AGN STORM paper viII submitted; Dehghanian et al. 2019, AGN STORM, paper x, in press.

An obvious consequence of a weaker and softer EUV continuum is that if the same gas responsible for producing the broad emission lines also emits a significant diffuse continuum component, as described in KG01, then a reduction in the flux of ionising photons incident upon BLR gas will result in a reduction in the production of both emission lines and diffuse continuum. Identification of the anomalous behaviour in the continuum bands therefore provides corroborating evidence for a significant variable diffuse continuum component arising from BLR gas, and furthermore, affords a means of estimating its overall contribution. In particular, this represents the strongest observational evidence yet of the influence of the variable diffuse continuum at wavelengths outside of the Balmer jump region. While its influence in the vicinity of the Balmer jump was suggested by the excess lag in $u^{\prime}$-band and made clear by the HST lag spectrum of NGC 4593, the presence of the diffuse continuum from the BLR at longer wavelengths was mainly inferred (Cackett et al. 2018). From the point of view of understanding the central accretion region of AGN, the variable diffuse continuum from BLR gas represents a nuisance component which must be accounted for in disk and broad emissionline reverberation mapping experiments, and is particularly problematic in the longer wavelength optical/near-IR continuum bands, where the delays and flux contributions are expected to be generally larger (KG01).

Over the range of measured UV-optical continuum bands, the diffuse continuum is expected to contribute minimally near the $\lambda 1157 \AA$ continuum, and generally increases in contribution toward longer wavelengths, reaching local maxima near the Balmer and Paschen thresholds. The larger 


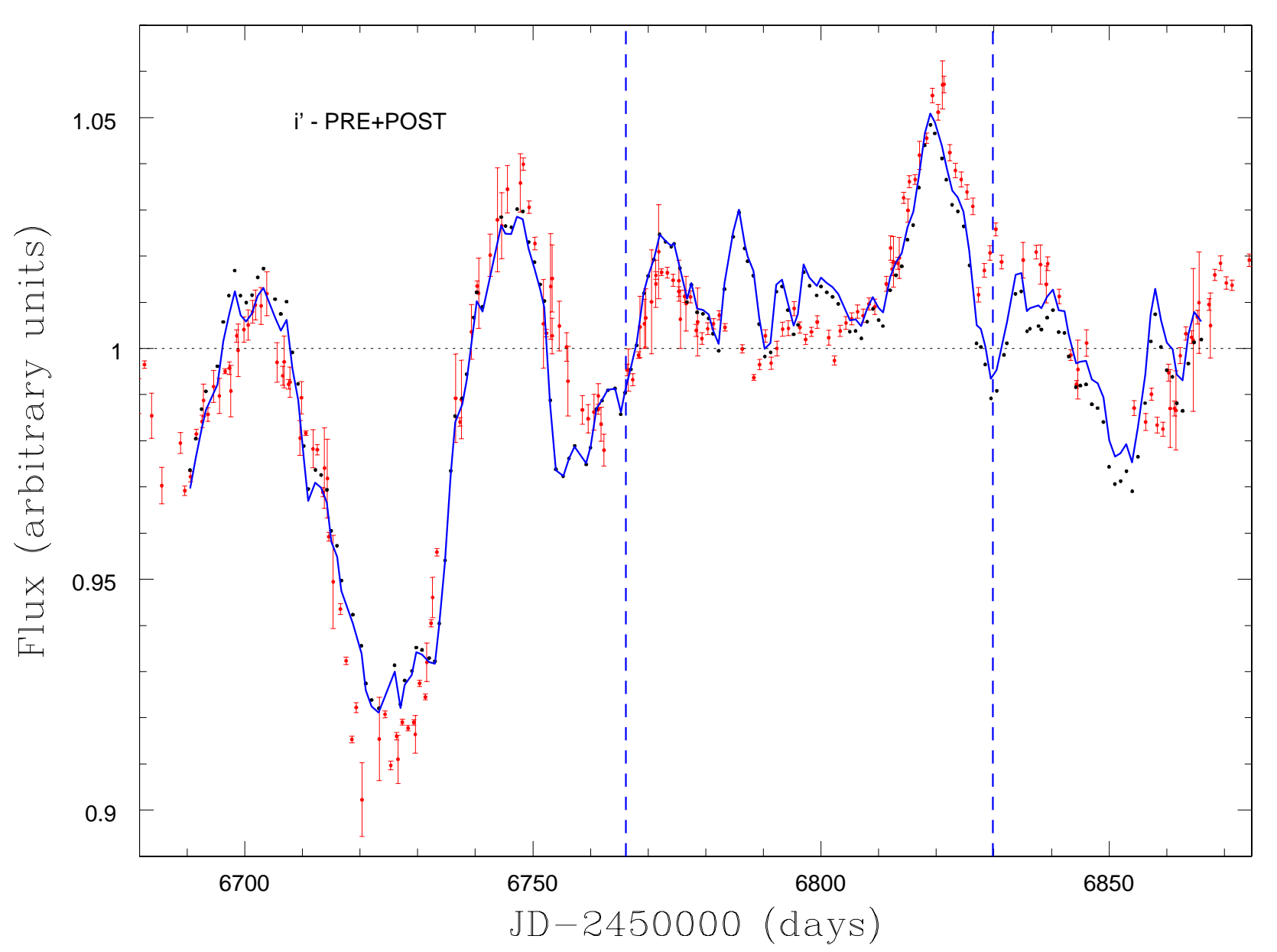

Figure 8. Ground-based i' broadband continuum light curve (red points), matched to a shifted, scaled and smoothed version of the $H S T$ $1157 \AA$ continuum band light curve (black points). The solid blue-line indicates the best-fit model obtained when an additional linear background component is included (see text for details). The dashed blue vertical lines indicate the time period spanning the anomalous behaviour of the broad emission-lines reported by Goad et al. (2016) (epochs JD 2456766.1-2456829.8). This region is excluded from the fit statistic. Lower-panel - the time variation of the $\chi^{2}$ statistic.

the contribution, the smaller the inter-band correlation is expected to be. If this nuisance component is ignored, the larger measured delays will lead to an overestimate of the "size" of the accretion disk, as has been found from several recent intensive disk reverberation mapping campaigns of nearby AGN, including NGC 5548 (Edelson et al. 2015; Fausnaugh et al. 2016; Edelson et al. 2017; Cackett et al. 2018). Furthermore, significant contamination of the optical continuum band by BLR diffuse continuum emission will lead to an underestimate of the BLR size in optical RM campaigns, over and above that already imposed by an intrinsically delayed optical continuum arising from the spatially extended disk.

Models that attempt to explain all of the inter-band $U V$-optical continuum variations, without including the expected contribution from BLR gas, are very likely flawed. For example, Gardner \& Done (2017) attribute all of the continuum inter-band delays to a second reprocessing re- gion, a puffed up Comptonised region, which they refer to as the EUV torus. This EUV torus reprocesses hard X-ray emission into lower amplitude more slowly varying EUV radiation, which in turn, illuminates the outer disk. In their model the longer wavelength UV-optical continuum interband delays are not dominated by light travel-time effects. Rather, they originate within the outer regions of their proposed EUV torus, and are related to changes in scale height and size as the Comptonised disk expands and contracts in response to X-ray heating at its inner edge. While they dismiss contamination of the broad-band UV and optical continuum by broad emission lines (as already demonstrated by Fausnaugh et al. 2016), missing from their model is the significant and variable continuum emission from the BLR. Indeed, Fausnaugh et al. (2016) showed that the enhanced delays in the longer wavelength $R$ and $r^{\prime}$ bands could reasonably be explained in terms of contaminating broad $\mathrm{H} \alpha$ $(\approx 20 \%$ and $\approx 15 \%$ respectively $)$, while those in the vicinity 
of the little blue bump ( $U$ and $u^{\prime}$ ) could be attributed to a significant $(\approx 19 \%)$ diffuse continuum component from BLR gas with similar delays as the Balmer lines.

The substantial drops in delay longward of the Balmer and Paschen jumps, a strong prediction of photoionisation model calculations and a key diagnostic of a significant diffuse continuum component (KG01; Lawther et al. 2018), is clearly visible in their data (Edelson et al. 2015; Fausnaugh et al. 2016). The best evidence to date for significant Balmer and Paschen jumps are seen in the intensive multi-wavelength spectro-photometric monitoring data for NGC 4593 (Cackett et al. 2018). Indeed, McHardy et al. (2018) identify the extended tails in the response functions for NGC 4593 with reprocessed continuum emission from the surrounding gas (i.e., the BLR).

Starkey et al. (2017) simultaneously fit a linearised $\alpha$ disk model to the $19 \mathrm{HST} /$ Swift and ground-based continuum data taken as part of the AGN STORM monitoring campaign on NGC 5548 . They find that the standard $\alpha$-disk model $T(r) \propto r^{-\alpha}$, with $\alpha$ fixed at 3/4, is a reasonably good fit to the UV-optical continuum light-curves, except for $u^{\prime}$, where the model tends to lead the data and is more highly variable. To fit the model, they require consistently larger rescaling of the error bars at longer wavelengths, which may indicate that the longer wavelength variations cannot adequately be described by a simple linearised echo model. These findings we argue are further evidence for contamination of the delay signal by diffuse continuum originating in the BLR.

In summary, the detection of the anomaly in the UV and optical continuum bands, coupled with the increased delays over and above that expected from a simple disk reprocessing scenario, and the presence of significant delay changes either side of the Balmer and Paschen jumps, we suggest provides the first clear evidence of a significant contribution to the UV-optical continuum from broad emission line region gas.

\section{CONCLUSIONS}

A standard assumption in RM is that the observed continuum, and in particular the far-UV continuum, is a suitable proxy for the driving continuum at ionising energies. However, during the 2014 AGN STORM monitoring campaign of NGC 5548, this assumption was violated, as evidenced by the decorrelation between the strong UV broad emission lines and the shortest accessible UV continuum band approximately mid-way through the campaign, here referred to as the anomalous period. However, we have shown that by using key diagnostic emission lines (Ly $\alpha$ and He II) we can infer a proxy for the variable driving ionising continuum incident upon BLR clouds. For NGC 5548, these key diagnostic emission lines indicate a weakening and softening of the EUV continuum ( $\gtrsim 30 \%$ in the Lyman continuum and $\gtrsim 40 \%$ in the 4 Rydberg continuum) relative to the shortest available HST/COS continuum band $(\lambda 1157 \AA)$ during the period of anomalous behaviour exhibited by the strong broad UV and optical emission lines. In addition, most of the ionising continuum was apparently much less variable than was the UV continuum during the anomalous period. The derivation of an appropriate proxy for the behaviour of the time-variable driving ionising continuum is crucial first step towards the recovery of the broad emission-line response functions in this source.

We next compared the level of correlation between the $\lambda 1157$ continuum fluxes and the fluxes within all other UV-optical continuum bands, both outside and during the anomalous period. We found a general trend that the Spearman rank correlation coefficient diminished with increasing wavelength of the continuum band, but also that the correlation during the anomalous period was significantly depressed relative to that outside this time period, particularly within wavelength bands where we expect the contribution from the continuum emanating from the BLR is greater. This is what we would expect if the BLR is contributing to the continuum flux measurements and if the BLR saw a weaker and less variable ionising EUV continuum during the anomalous period. We also compared scaled, smoothed, and timedelayed versions of the $\lambda 1157$ continuum light curve to each of the other UV-optical continuum bins as another means of exploring the likely decorrelation of the EUV continuum from the observed UV-optical continuum, using the potential presence of the BLR diffuse continuum within the continuum bands as a marker of this decorrelation in flux and variability.

Importantly, we find corroborative evidence for anomalous behaviour in virtually all of the longer wavelength (relative to $\lambda 1157 \AA$ ) UV and optical continuum bands. This we suggest indicates a significant contribution to the variable UV-optical continuum emission from the diffuse continuum emitted by BLR clouds. This has a direct impact on the physical interpretation of the measured inter-band continuum delays, and by inference, the size and temperature dependence of the accretion disk.

\section{REFERENCES}

Baldwin, J., Ferland, G., Korista, K., \& Verner, D. 1995, ApJ 455, 119 .

Barth, A.J., Pancoast, A., Thorman, S.J., Bennert, V.N., Sand, D.J. et al. 2011, ApJ 743, 4.

Barth, A.J., Pancoast, A., Bennert, V.N., Brewer, B.J., Canalizo, G. et al. 2013, ApJ 769, 128.

Bentz, M. C., Walsh, J. L., Barth, A. J., et al. 2010a, ApJ 716, 993

Bentz, M. C., Horne, K.D., Barth, A. J., et al. 2010b, ApJ 720, 46

Bentz, M. C., Horenstein, D., Bazhaw, C., et al. 2014, ApJ 796, 8

Bentz, M. C., \& Katz, S. 2015, PASP 127, 67

Bentz, M. C., Walsh, J. L., Barth, A. J., et al. 2009, ApJ 697, 160 Blandford, R.D., \& McKee, C.F. 1982, ApJ 255, 419.

Cackett, E.M., Chiang, C-Y., McHardy, I., et al. 2018, ApJ 857, 53 .

Cackett, E.M., Horne, K.D., \& Winkler, H. 2007, MNRAS 380, 669.

Clavel, J., Wamsteker, W., \& Glass, I.S. 1989, ApJ 337, 236.

Clavel, J., Reichert, G.A., Alloin, D., Crenshaw, D.M., Kriss, G.A. et al. 1991, ApJ 366, 64 .

Collier, S. 2001, MNRAS 3251527.

Collier, S., \& Peterson, B.M. 2001, ApJ 555775.

Dehghanian, M., Ferland, G.J., Kriss, G.A., Peterson, B.M., Mathur, S. et al. 2019, ApJ in press, arXiv:1812.11578.

Denney, K.D., Peterson, B.M., Pogge, R.W., Adair, A., Atlee, D.W. et al. 2009, ApJ 704, 80. 
De Rosa, G., Peterson, B.M., Ely, J., et al. 2015, ApJ 806, 128, (Paper I).

Dexter, J., \& Agol, E. 2011, ApJ 727, 24.

Edelson, R., Gelbord, J.M., Horne, K.D., et al. 2015, ApJ 806, 129, (Paper II).

Edelson, R., Gelbord, J., Cackett, E., Connolly, S., Done, C., et al. 2017, ApJ 840, 41.

Edelson, R., Gelbord, J., Cackett, E., Peterson, B.M., Horne, K. et al. 2018, ApJ in press, arXiv:1811.07956

Fausnaugh, M.M., Denney, K.D., Barth, A.J., Bentz, M.C., Bottorff, M.C. et al. 2016, ApJ 821, 56, ( Paper III).

Gardner, E., \& Done, C. 2017, MNRAS 470, 3591.

Goad, M.R., Korista, K.T., \& Ruff, A.J. 2012, MNRAS 426, 3086.

Goad, M.R., \& Korista, K.T. 2014, MNRAS 444, 43.

Goad, M.R., Korista, K.T., De Rosa, G., Kriss, G.A., Edelson, R., et al. 2016, ApJ 824, 11, (Paper IV).

Grier, C.J., Peterson, B.M., Pogge, R.W., et al. 2012, ApJ 755, 60.

Hall, P.B., Sarrouh, G.T., \& Horne, K.D. 2018, ApJ 854, 93.

Horne, K.D., Welsh, W.F., \& Peterson, B.M. 1991, ApJ 367, 5.

Korista, K.T., Baldwin, J.A., \& Ferland, G.J. 1998 ApJ 507, 24.

Korista, K.T., \& Ferland, G.J. 1998 ApJ 495, 672.

Korista, K.T., \& Goad, M.R. 2001, ApJ 553, 695 (KG01).

Koshida, S., Minezaki, T., Yoshii, Y., Sugawara, S., Enya, K., et al. 2014, ApJ 788, 159.

Kriss, G.A., et al. ApJ in press, (Paper VIII).

Krolik, J.H., Horne, K.D. Kallman, T.R., Malkan, M.A., Edelson, R.A., \& Kriss, G.A. 1991, ApJ 371, 541.

Lawther, D., Goad, M.R., Korista, K.T., Ulrich, O., \& Vestergaard, M. 2018, MNRAS 481, 533.

Lu, K-X., Du, P., Hu, C. Li, Y-R., Zhang, Z-H., et al. 2016, ApJ $827,118$.

Mathur, S., Gupta, A., Page, K., et al. 2017, ApJ 846, 55.

McHardy, I.M., Connolly, S.D., Horne, K.D., Cackett, E.M., Gelbord, J. et al. 2018, MNRAS 480, 2881.

Minezaki, T., Yoshii, Y., Kobayashi, Y., Enya, K., Suganuma, M., et al. 2004, ApJ 600, 35.

Morgan, C.W., Kochanek, C.S., Morgan, N.D., \& Falco, E.E. 2010, ApJ 712, 1129.

Mosquera, A.M., Kochanek, C.S., Chen, B., et al. 2013, ApJ 769, 53.

Narayan, R. 1996, ApJ 462, 136.

Nealon, R., Price, D.J., \& Nixon, C.J. 2015, MNRAS 448, 1526.

Oknyanskij, V.J., \& Horne, K.D. 2001, ASPC 224, 149.

Pancoast, A., Brewer, B.J., \& Treu, T. 2014, MNRAS 455, 3055.

Pancoast, A., Brewer, B.J., Treu, T., et al. 2012, ApJ 754, 49

Pancoast, A., Brewer, B.J., Treu, T., et al. 2014, MNRAS 445, 3073

Pei, L., Fausnaugh, M.M., Barth, A.J., Peterson, B.M., Bentz, M., et al. 2017, ApJ 837, 131, (Paper v).

Peterson, B.M., Berlind, P., Bertram, R., et al. 2002, ApJ 581, 197

Peterson, B.M. 2014, Space Sci. Rev. 183, 253

Poindexter, S., Morgan, N., \& Kochanek, C.S. 2008, ApJ 673, 34 Sergeev, S.G., Doroshenko, V.T., Golunbinskiy, Y.V., Merkulova, N.I., \& Sergeeva, E.A. 2005, ApJ 622, 129.

Shakura, N.I., \& Sunyaev, R.A. 1973, A\&A 24, 337.

Silk, J., \& Rees, M.J. 1998, AAP 331, L1-L4.

Silk, J. 2013, ApJ 772, 112.

Suganuma, M., Yoshii, Y., Kobayashi, Y., Minezaki, T., Enya, K., et al. 2006, ApJ 639, 46.

Starkey, D., Horne, K.D., Fausnaugh, M.M., Peterson, B.M., Bentz, M.C., et al. 2017, ApJ 835, 65, (Paper VI).

Sun, M., Xue, Y., Cai, Z., \& Guo, H. 2018, ApJ 857, 86.

Troyer, J., Starkey, D., Cackett, E.M., Bentz, M., Goad, M.R., et al. 2016, MNRAS 456, 4040.

Wamsteker, W., Rodriguez-Pascual, P., Wills, B.J., Netzer, H., Wills, D., et al. 1990, ApJ 354, 446.
Welsh, W.F., \& Horne, K.D. 1991, ApJ 379, 586.

This paper has been typeset from a $\mathrm{TEX}_{\mathrm{E}} / \mathrm{LATEX}$ file prepared by the author. 